\title{
Human limb skeletal muscle wasting and architectural remodeling during five to ten days intubation and ventilation in critical care - an observational study using ultrasound
}

Peter Turton ${ }^{1 *}$ (D), Richard Hay', Jonathon Taylor ${ }^{2}$, Jamie McPhee ${ }^{3}$ and Ingeborg Welters ${ }^{4}$

\begin{abstract}
Background: Critically ill patients frequently suffer muscle weakness whilst in critical care. Ultrasound can reliably track loss of muscle size, but also quantifies the arrangement of the muscle fascicles, known as the muscle architecture. We sought to measure both pennation angle and fascicle length, as well as tracking changes in muscle thickness in a population of critically ill patients.

Methods: On days 1,5 and 10 after admission to critical care, muscle thickness was measured in ventilated critically ill patients using bedside ultrasound. Elbow flexor compartment, medial head of gastrocnemius and vastus lateralis muscle were investigated. In the lower limb, we determined the pennation angle to derive the fascicle length.

Results: We recruited and scanned 22 patients on day 1 after admission to critical care, 16 were re-scanned on day 5 and 9 on day 10. We found no changes to the size of the elbow flexor compartment over 10 days of admission. In the gastrocnemius, there were no significant changes to muscle thickness or pennation angle over 5 or 10 days. In the vastus lateralis, we found significant losses in both muscle thickness and pennation angle on day 5, but found that fascicle length is unchanged. Loss of muscle on day 5 was related to decreases in pennation angle. In both lower limb muscles, a positive relationship was observed between the pennation angle on day 1 , and the percentage of angle lost by days 5 and 10 .

Discussion: Muscle loss in critically ill patients preferentially affects the lower limb, possibly due to the lower limb becoming prone to disuse atrophy. Muscle architecture of the thigh changes in the first 5 days of admission, in particular, we have demonstrated a correlation between muscle thickness and pennation angle. It is hypothesised that weakness in the lower limb occurs through loss of force generation via a reduced pennation angle.
\end{abstract}

Conclusion: Using ultrasound, we have been able to demonstrate that muscle thickness and architecture of vastus lateralis undergo rapid changes during the early phase of admission to a critical care environment.

Keywords: Muscle wasting, Weakness, Critical care, Ultrasound, Muscle architecture, Pennation angle

\footnotetext{
*Correspondence: peterturton@doctors.net.uk

${ }^{1}$ Intensive Care Unit, Royal Liverpool University Hospital, Prescot Street,

Liverpool L7 8XP, UK

Full list of author information is available at the end of the article
}

\section{$\int$ Biomed Central}

(c) 2016 The Author(s). Open Access This article is distributed under the terms of the Creative Commons Attribution 4.0 International License (http://creativecommons.org/licenses/by/4.0/), which permits unrestricted use, distribution, and reproduction in any medium, provided you give appropriate credit to the original author(s) and the source, provide a link to the Creative Commons license, and indicate if changes were made. The Creative Commons Public Domain Dedication waiver (http://creativecommons.org/publicdomain/zero/1.0/) applies to the data made available in this article, unless otherwise stated. 


\section{Background}

Intensive Care Unit Acquired Weakness (ICUAW) is a common consequence of prolonged stay in the intensive care setting. Generalised muscle weakness due to muscle wasting is a key feature of the disease [1]. As a result, survivors of intensive care admission have difficulty with mobility and require complex rehabilitation [2, 3].

Previous work on ICUAW has identified many interrelated factors that put patients at risk of muscle wasting, including increased protein degradation, the use of steroids, high levels of circulating pro-inflammatory mediators and the presence of multi organ failure [4, 5]. The prolonged immobility of intubated and ventilated patients has also been suggested to be a significant risk factor [6].

Ultrasound is increasingly being used to assess both cross sectional area and muscle thickness at the bedside of the patient, and is a useful tool in monitoring changes in muscle size over time [7]. Furthermore, when predicting adverse outcome in the intensive care environment, ultrasound assessment of the rectus femoris muscle has been shown to compare favourably with measures of frailty [8]. In studies performed in healthy subjects, measurements of muscle thickness and fascicle arrangement of the muscle using ultrasound are reproducible and are not dependent on age [9]. In the intensive care population, significant reductions in the cross sectional area of the rectus femoris muscle over a 10 day admission period have been demonstrated [10]. Muscle thickness of the quadriceps femoris has also been shown to decrease over 28 days, with muscle thickness also being negatively correlated with the length of stay [11].

As well as muscle thickness and cross sectional area, ultrasound can also be used to visualise the arrangement of the fascicles within the muscle, which informs about both the pennation angle and the length of the fascicle.

It has been shown in the triceps brachii that muscle thickness is positively correlated with pennation angle [12] and further, pennation angle is positively correlated with the cross sectional area of vastus lateralis [13] and rectus femoris [14]. Muscle force has been suggested to increase with increasing pennation angle, up to a limit of 45 degrees [15]. The same paper found that in healthy subjects, resistance based strength training was associated with an increased cross sectional area, pennation angle and contractile strength, suggesting an interrelation between muscle architecture variables and force generation. However, pennation angle has never been used to assess muscle wasting in critically ill patients.

In this study, we aimed to establish changes in pennation angle and fascicle length as markers of altered fascicle arrangement in critical illness. We furthermore wanted to compare muscle wasting in areas of the upper and the lower limb by assessing muscle thickness of the vastus lateralis muscle, the medial head of gastrocnemius muscle and the elbow flexor compartment in ventilated patients.

\section{Methods \\ Participants}

The study received ethical approval from the Local Research Ethics Committee (UK NHS Health Research Authority, Wales Research Ethics Committee 4 (REC 4) Wrexham, Wales, United Kingdom, approval number 13/WA/0111). Patients were recruited from the Intensive Care Units of the Royal Liverpool University Hospital, or Warrington District General Hospital over a 1 year study period. Informed assent was obtained from the patients' next of kin, and where possible, retrospective consent was sought when the patients had regained capacity.

Patients who were greater than 18 years of age who were assented within $24 \mathrm{~h}$ of being intubated and admitted to the participating intensive care units were included in the study. Exclusion criteria included being under 18 years of age pregnancy, trauma to either the right lower or right upper limbs, history of neurological, neuromuscular or muscular wasting diseases, rhabdomyolysis, vascular insufficiency or amputation of the right upper or lower limbs, and prolonged immobility prior to admission to intensive care.

\section{Ultrasound scanning}

On day 1 of imaging, all patients were intubated, ventilated and sedated. Imaging on subsequent days was performed in patients who were still intubated and ventilated, but may not have been sedated, according to their clinical progress. None of the patients were given continuous muscle relaxant infusions on the day of scanning.

Patients had ultrasound scans of the right elbow flexor compartment (Fig. 1a), right medial head of gastrocnemius (Fig. 1b) and right vastus lateralis (Fig. 1c) muscles were performed within $24 \mathrm{~h}$ of intubation. Ultrasound scans were undertaken with B-mode ultrasonography, using a linear array probe. One of three trained operators performed the ultrasound scans, and it was ensured that when an operator scanned a participant, the same researcher performed the repeat scans on days 5 and 10 . Patients were positioned in the supine anatomical position for ultrasound scanning, using a method previously described in a study of healthy volunteers undergoing prolonged bedrest [16].

Imaging of the right elbow flexor compartment was undertaken with the patient sat up at 45 degrees and the limbs positioned in the anatomical position, ensuring there was no flexion at the elbow. The ultrasound transducer was placed $5 \mathrm{~cm}$ proximal to the line connecting 

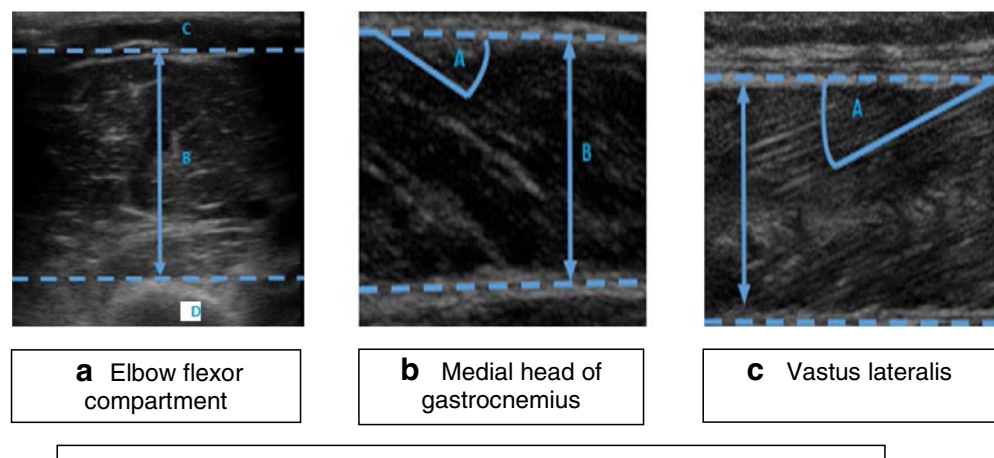

C Vastus lateralis

Key $-\mathrm{A}=$ measured pennation angle. $\mathrm{B}=$ Muscle thickness $\mathrm{C}=$ subcutaneous fat. $\mathrm{D}=$ Humerus

Fig. 1 Representative images of elbow flexor compartment (a), gastrocnemius (b) and vastus lateralis (c).

both the medial and lateral epicondyles, above the base of the ante cubical triangle and was positioned perpendicular to the plane of the muscle, to image the muscle thickness from the edge of muscle down to the tip of the humerus. Scanning in plane with the muscle was avoided, as in the upper limb, fascicles run parallel with the aponeuroses, making calculation of fascicle length inaccurate.

Muscle thickness measurement was made by firstly measuring the overall width seen on the image, from the top of the muscle down to the bottom of the image. Secondly, the distance from the tip of the humerus to the bottom of the image was measured and subtracted from the overall thickness to give the thickness of the muscle from humerus to the superior muscle edge.

Six images were taken per patient, the highest and lowest muscle width was removed, and the average of the four remaining images was used to give the final measurement for each patient.

For scanning of the vastus lateralis, patients remained sat at 45 degrees, but with the lower limb flat to the bed. The limb was then externally rotated to ensure the probe handle could be placed parallel to the ground. The probe was placed $10 \mathrm{~cm}$ proximal to the lateral condyle of the femur, in plane with the muscle to image both the superficial and deep aponeuroses of the vastus lateralis, and the muscle fascicles within the muscle. Imaging of the medial gastrocnemius was achieved by flexion at the knee to ensure the thigh was at 90 degrees to the tibia, with the foot placed flat onto the patient's bed. This is different to method used in healthy volunteers [16], but due to the inflatable mattresses used, this method exposes the medial head to enable imaging, again with the probe handle parallel to the ground. The probe was again placed in plane with the muscle, at the widest point in the muscle belly. Depth of scanning was altered until both the superficial and deep aponeuroses were visible, along with the fascicles within the muscle.
In both lower limb muscles, six images were taken. Muscle thickness (MT) was measured at the widest point in each image, from the superficial to the deep aponeuroses. Pennation angle (PA) was measured by measuring the angle in degrees between the fascicle and the superficial aponeurosis. This method measures the fascicle closest to the widest point in the muscle to minimise variation in pennation angle in just one muscle [17]. Fascicle length (FL) was derived from pennation angle and muscle thickness as described before $[18,19]$ '. Briefly, the following formula was used to derive fascicle length:

$$
\mathrm{FL}=\mathrm{MT} /(\sin \mathrm{PA})
$$

Muscle thickness was measured in pixels initially, before converting into centimetres, as each image had a scale of known depth running down the right hand side of the image, which always consisted of 380 pixels in depth. To prevent compression of the muscle during imaging, the ultrasound probe was coated with a thick layer of a water-based gel.

Once all six images were taken from each muscle, the images with the longest and shortest fascicle length were removed, and the average muscle width, pennation angle and fascicle length were calculated from the remaining four images. Image measurement was undertaken using ImageJ software (version 1.47, National Institutes of Health, USA).

Imaging was repeated on days 5 and 10, provided the patient remained intubated. Patients were not scanned again if they were extubated or had died prior to their next scheduled scan.

\section{Statistics}

Muscle thickness, pennation angle and fascicle length have been assessed using Friedman's test. Pairwise Friedman's tests were performed where results were 
significant (see Table 3). Significance has also been tested by constructing $95 \%$ confidence intervals for absolute changes to muscle thickness and pennation angle at both days 5 and 10, with intervals not passing through zero considered to be statistically significant.

Where correlations were tested, Pearson's product moment correlation coefficient was used, with a $p$-value of less than 0.05 being considered statistically significant. Changes in demographic data over time were assessed used a one-way ANOVA, with a Bonferroni correction applied where significant differences were found. Data was analysed using SPSS version 21 for Windows (IBM, USA).

\section{Results}

\section{Patient demographics}

Twenty two patients were recruited into the study and imaged on day 1 . Of these, 16 patients were scanned on day 5 , and 9 were scanned on day 10 . Patients who were not scanned on day 5 or 10 were either successfully extubated or died before their next scan (Fig. 2). Table 1 summarises the major patient demographics. There were no significant changes to age, weight, height or body mass index (BMI) during the study period. All patients met the criteria for SIRS, but not all had a diagnosis of sepsis (Table 2).

\section{Changes in muscle thickness}

A summary of measurements in all three muscles (Vastus lateralis muscle, medial head of gastrocnemius muscle, elbow flexor compartment) is presented in Table 3. Baseline measurements were performed on day 1. A significant correlation between muscle

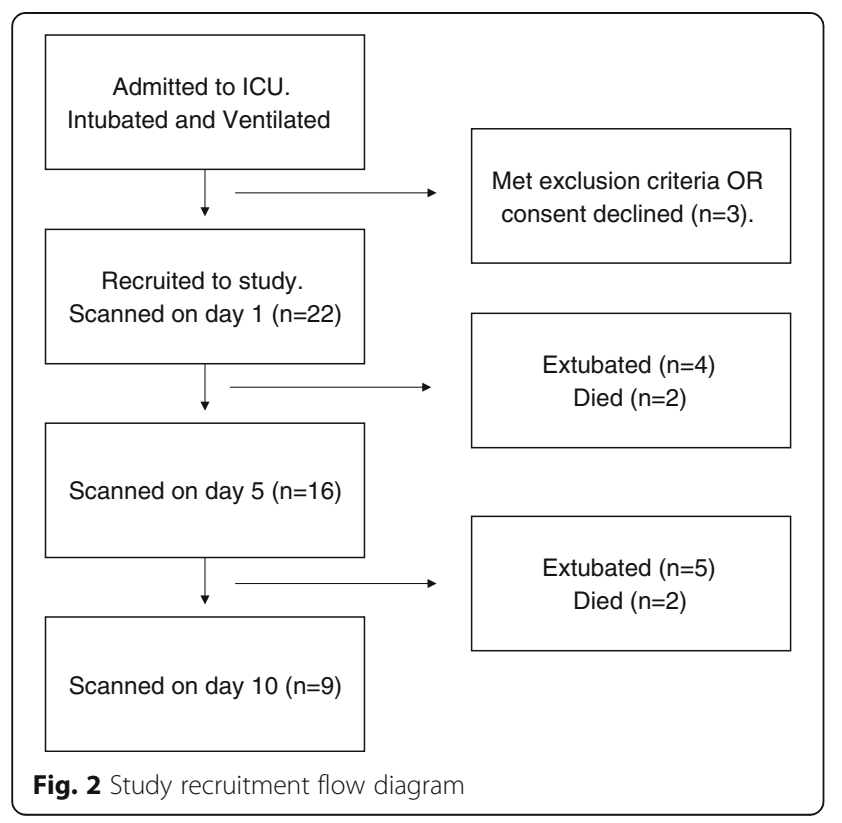

thickness in the upper and lower limb was demonstrated (Table 4).

\section{Elbow flexor compartment (Fig. 1a)}

On day 1 , there was a significant negative correlation between age and muscle thickness $(r=-0.39, p=0.03, n=$ $22)$. Height and weight were both significantly positively correlated with muscle thickness (height $r=0.41, p=$ 0.04 ; weight $r=0.57, p=0.006$ ).

There were no significant changes in muscle thickness in the elbow flexor compartment on either day 5 or day 10 , when compared to day 1 . Mean change in muscle thickness on day 5 was $2.62 \%$ (95\% CI -2.12 to $7.36 \%$, $p=0.26)$ and on day 10 was $3.79 \%$ (95\% CI: -15.70 to $23.28 \%, p=0.66)$. Age, weight, height and APACHE II score on admission did not correlate with absolute or relative change in muscle thickness.

\section{Medial head of gastrocnemius muscle (Fig. 1b)}

There were no significant changes in muscle thickness of the medial head of the gastrocnemius muscle on either day 5 or 10 . On day 5 , mean change in muscle thickness was $-5.08 \%$ (95\% CI: $-14.03 \%$ to $3.88 \%$, $p=0.25$ ), and on day 10 , the mean change in muscle thickness was $-15.69 \% \quad(95 \%$ CI: $-43.43 \%$ to $12.05 \%, p=0.23$ ).

Muscle thickness on day 1 was negatively correlated with absolute change in muscle thickness on day $5(r=$ $-0.53, p=0.02)$ and day $10(r=-0.662, p=0.03)$.

\section{Vastus lateralis (Fig. 1c)}

In the vastus lateralis muscle, age was negatively associated with muscle thickness $(r=-0.54, p=0.007)$ and fascicle length $(r=-0.53, p=0.007)$. Further, patient weight was positively associated with muscle thickness $(r=0.41$, $p=0.04)$.

There were significant losses of both muscle thickness over both 5 and 10 days; a mean change of $-10.66 \%$ (95\% CI: -17.48 to $-3.83 \%, p=0.005$ ) was seen on day 5 , a mean change of $-28.81 \%$ (95\% CI: -37.39 to $-20.23 \%$, $p<0.0001$ ) on day 10, see Fig. 4a.

Muscle thickness on day 1 was not significantly correlated with absolute change in muscle thickness on day 5 $(r=-0.43, p=0.05)$ but this correlation was observed by day $10(r=-0.770, p=008)$.

\section{Changes in lower limb muscle architecture Medial head of gastrocnemius muscle}

In both the medial head of gastrocnemius and vastus lateralis muscles, no relationship was observed between the changes in muscle architecture and age, weight, height, BMI or APACHE II score.

On day 1, muscle thickness of gastrocnemius muscle was positively correlated with pennation angle $(r=0.41$, 
Table 1 Patient characteristics

\begin{tabular}{lllll}
\hline & Day 1 & Day 5 & Day 10 & $P$-value \\
\hline Sample Size & 22 & 16 & 9 & - \\
Males, n (\%) & $19(86.3)^{*}$ & $13(81.3)$ & $7(77.7)$ & $58.66 \pm 19.95$ \\
Age, [years] (Mean \pm SD) & $59.77 \pm 16.74$ & $63.12 \pm 16.44$ & $1.71 \pm 0.13$ & 0.001 \\
Height, [metres] (Mean \pm SD) & $1.72 \pm 0.13$ & $1.68 \pm 0.13$ & $75.66 \pm 24.58$ & 0.78 \\
Weight, [kg] (Mean \pm SD) & $88.50 \pm 29.19$ & $82.95 \pm 27.31$ & $25.73 \pm 7.40$ & 0.54 \\
Body Mass Index, (Mean \pm SD) & $30.75 \pm 12.50$ & $30.99 \pm 13.69$ & 0.61 \\
\hline
\end{tabular}

Data tested with one-way ANOVA with Bonferroni correction applied. Statistically significant $p$-values $(p<0.05)$ have been marked with ${ }^{*}$. Despite patients withdrawing throughout the study period, there were no significant changes to group's demographic variables. The number of males compared to females on day 1 was compared using the binomial test

$p=0.03, n=21)$, and positively correlated with fascicle length on day $1(r=0.60, p=0.002)$. Pennation angle was negatively associated with fascicle length on day 1 $(r=-0.43, p=0.028)$.

On day 5 , mean change in pennation angle was $0.92 \%$ (95 \% CI: -9.02 to $7.17 \%, p=0.81$ ), and mean change in fascicle length was $2.47 \%$ (95 \% CI: -13.11 to $8.15 \%$, $p=0.63)$. On day 10 , mean change in pennation angle was $-6.73 \%$ (95 \% CI: -23.13 to $9.66 \%, p=0.37$ ) and mean change in fascicle length was $-9.89 \%$ (95 \% CI: -31.45 to $11.67 \%, p=0.32$ ).

Pennation angle on day 1 was significantly negatively correlated with percentage change on day $5(r=-0.71, p=0.009$, $n=16)$ and day $10(r=-0.72, p=0.014, n=9$, see Fig. 3$)$.

\section{Vastus lateralis muscle}

For pennation angle, a mean change of -14.31 and $-28.47 \%$ was seen on days 5 and 10 respectively (Day 5 $95 \%$ CI: -25.53 to $-3.09, p=0.006$; Day 10: -41.98 to $-14.95, p=0.001$, see Fig. $4 \mathrm{~b})$. There were no significant changes to fascicle length on either day.

Pennation angle on day 1 was significantly negatively associated with mean change in angle on day 5 $(r=-0.43, p=0.04$, see Fig. 5).

Mean change in muscle width on day 5 was significantly positively correlated with mean change in pennation angle on day $5(r=0.78, p<0.001$, see Fig. 6$)$.

\section{Discussion}

In this study we have demonstrated a loss in muscle thickness and a decrease in pennation angle in the

Table 2 Patient characteristics by presenting diagnosis

\begin{tabular}{ll}
\hline Catergory of diagnosis & Number of patients, N, (\%) \\
\hline Sepsis & $11(50 \%)$ \\
Post surgery & $4(18 \%)$ \\
Post cardiac arrest & $4(18 \%)$ \\
Overdose & $3(9 \%)$ \\
\hline
\end{tabular}

All patients met the criteria for Systemic Inflammatory Response Syndrome (SIRS) vastus lateralis. These results support previous studies demonstrating that admission to intensive care causes a reduction in muscle size in the thigh $[10,11]$, with of loss of muscle thickness in the vastus lateralis muscle occurring within the first 5 days of admission. In our study, muscle wasting occurred earlier than reported in the literature: a previous study found that the cross sectional area of the rectus femoris muscle significantly decreased in the first 7 days of admission, whilst showing no changes in the first 3 days [10]. Further loss of muscle thickness has also been related with loss of muscle function on discharge from critical care [20].

In our study, this loss of muscle thickness in the thigh is coupled with a significant reduction in the pennation angle of the muscle fascicles, but maintaining fascicle length. The decline in pennation angle continues until day 10 post-intubation. In the medial gastrocnemius we have shown similar trends towards loss of both muscle thickness and pennation angle in the first $96 \mathrm{~h}$, however these results did not reach statistical significance. Large pennation angles are able to pack more contractile components in parallel into a certain volume, and can generate greater force up to angles of 45 degrees $[15,21]$. If loss of angle leads to a loss of force generation, then this could help to explain the weakness patients experience on being discharged from critical care. We further demonstrated that in both muscles, vastus lateralis and gastrocnemius, muscle thickness and pennation angle are related and support previous studies that thicker muscles could generate greater force by having larger pennation angles, and consequently, larger cross sectional areas [15] There was also a relationship in both muscles between the pennation angle on day 1 , and how much of that angle was lost by day 5 , suggesting that patients who had a larger pennation angle on day 1 lost a greater percentage of their pennation angle than patients who were admitted with smaller pennation angles. This relationship also held for muscle thickness, with muscle thickness decreasing to a greater degree in thicker muscles. This may indicate that muscles with larger pennation angles also have greater muscle thickness by having 
Table 3 Changes in measured variables in the elbow flexor compartment, gastrocnemius and vastus lateralis muscles

\begin{tabular}{|c|c|c|c|c|}
\hline & Day $1(n=22)$ & Day $5(n=16)$ & Day $10(n=9)$ & $P$-value \\
\hline $\begin{array}{l}\text { MT - Elbow flexor compartment, }[\mathrm{cm}] \\
\text { (median } \pm \text { IQR) }\end{array}$ & $3.20 \pm 0.58$ & $3.30 \pm 0.87$ & $2.98 \pm 0.83$ & 0.62 \\
\hline $\begin{array}{l}\text { MT - Gastrocnemius, [cm] } \\
\text { (median } \pm \text { IQR) }\end{array}$ & $1.29 \pm 0.60$ & $1.34 \pm 0.43$ & $1.14 \pm 0.65$ & 0.72 \\
\hline MT - Vastus lateralis [cm] (median \pm IQR) & $1.53 \pm 0.77$ & $1.40 \pm 0.46$ & $1.18 \pm 0.36$ & $0.002^{*}$ \\
\hline $\begin{array}{l}\mathrm{FL}-\text { Gastrocnemius }[\mathrm{cm}] \\
\text { (median } \pm \text { IQR) }\end{array}$ & $3.99 \pm 1.84$ & $3.69 \pm 1.37$ & $3.43 \pm 2.12$ & 0.90 \\
\hline $\begin{array}{l}\mathrm{FL}-\text { Vastus lateralis }[\mathrm{cm}] \\
\text { (median } \pm \mathrm{IQR})\end{array}$ & $8.1 \pm 3.06$ & $8.45 \pm 4.55$ & $7.85 \pm 5.89$ & 0.89 \\
\hline PA - Gastrocnemius [degrees] (median \pm IQR) & $20.93 \pm 6.41$ & $19.82 \pm 5.65$ & $17.5 \pm 3.91$ & 0.37 \\
\hline PA - Vastus lateralis [degrees] (median \pm IQR) & $11.09 \pm 4.88$ & $9.86 \pm 3.69$ & $8.03 \pm 3.86$ & $0.018^{*}$ \\
\hline
\end{tabular}

MT Muscle thickness, FL Fascicle length, PA Pennation angle

Significance tested with Friedman's test, followed by pairwise Friedman's tests where $p<0.05$. Stastically significant $p$-values have been marked with *. Muscle thickness and pennation angle of the vastus lateralis muscle significantly decreased over both 5 and 10 days

greater numbers of sarcomeres in parallel to the direction of the fascicle. It may well be that these parallel sarcomeres are lost first, causing loss of pennation angle, and consequently loss of muscle thickness; a recent review article has also suggested that physical training may increase muscle cross sectional area by increasing parallel addition of sacromeres, and are lost in the same way after a period of disuse [22]. The lack of reduction in fascicle length may suggest that serial sarcomeres are preserved.

Age-related deterioration of the pennation angle of both the vastus lateralis muscle [9] and medial gastrocnemius muscle [23] have previously been described, as well an age-related loss of muscle thickness [24]. To our knowledge, this is this first paper that describes how the muscle architecture of pennate muscles changes due to immobility through intubation and ventilation. This addresses previous criticism of a recent literature review describing that although ultrasound is used to measure muscle thickness and cross sectional area, the changes to muscle architecture have not been described yet [25].

Interestingly, the changes we describe above were strictly limited to the lower limb and could not be reproduced in elbow flexor compartment. The size of the elbow flexor compartment remained unchanged

Table 4 Comparison of muscle thickness between all three muscles on day 1

\begin{tabular}{llcl}
\hline Muscle 1 & Muscle 2 & $\begin{array}{c}\text { Correlation } \\
\text { coefficient, r }\end{array}$ & $P$-value \\
\hline Elbow flexor compartment & Vastus lateralis & 0.50 & $0.01^{*}$ \\
Elbow flexor compartment & $\begin{array}{l}\text { Medial head of } \\
\text { gastrocnemius }\end{array}$ & 0.54 & $0.006^{*}$ \\
Vastus lateralis & $\begin{array}{l}\text { Medial head of } \\
\text { gastrocnemius }\end{array}$ & 0.60 & $0.002^{*}$ \\
\hline
\end{tabular}

Pearson's product-moment correlation coefficient, ${ }^{*} p<0.05$ throughout the first 10 days of admission. These results are in line with a previous study in healthy volunteers undergoing a five week period of bed rest that had shown that muscle thickness decreased in the both the vastus lateralis and medial gastrocnemius, but not in the biceps brachii or tibialis anterior [16]. Our findings support the theory that in healthy and critically ill individuals, non-weight bearing muscles are less susceptible to wasting, compared to muscles which have a daily load placed up on them through the action of walking or standing.

Due to its force- generating properties, pennation angle should be monitored in all interventions aimed at preventing muscle wasting in critical illness. Both early mobilisation [26] and neuromuscular electrical stimulation [27] have been shown to prevent muscle atrophy in critical care patients, although their effects on muscle architecture have not yet been described and needs to be elucidated in further studies.

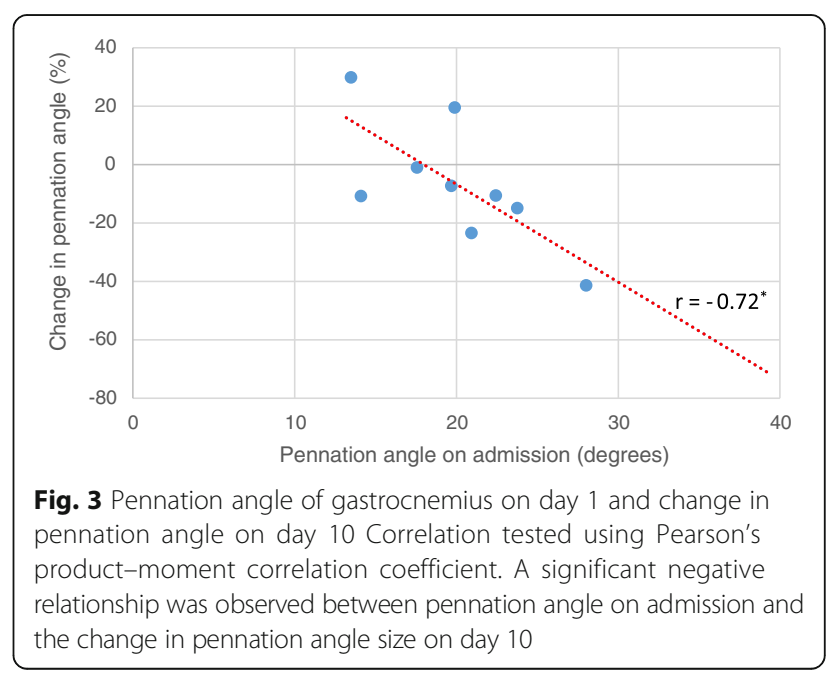




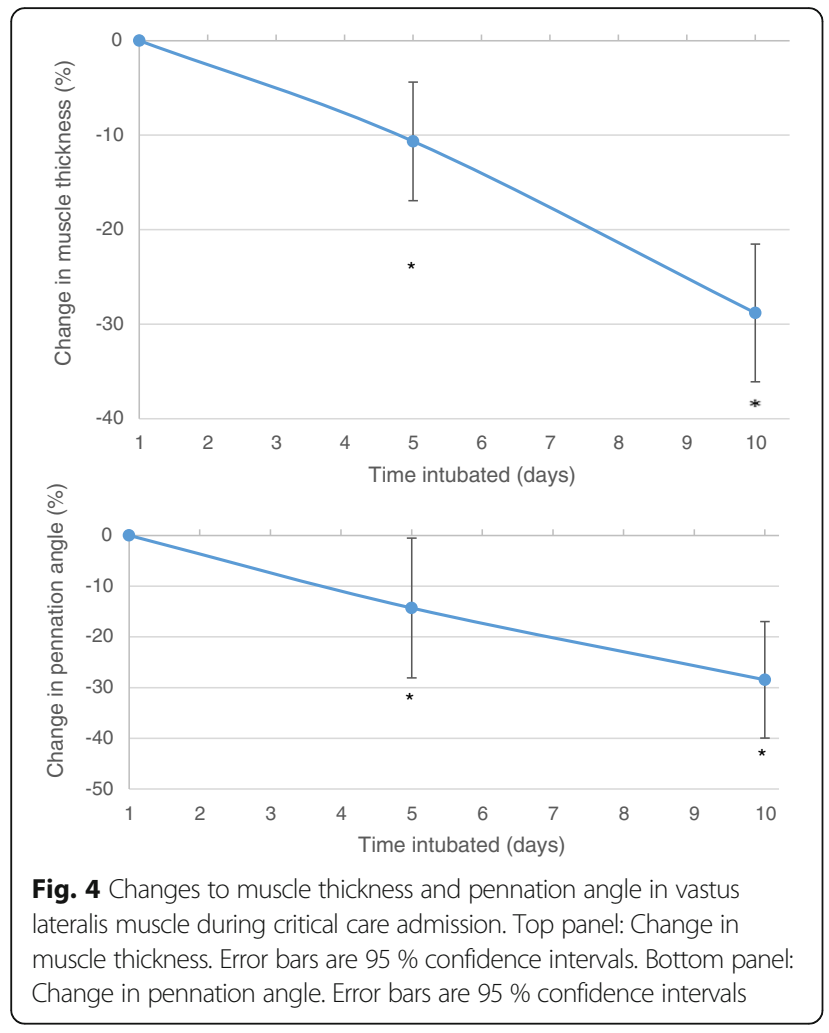

Our study is limited by several factors, in particular the small sample size and the heterogeneity of the patient cohort. There were significantly more males recruited compared to females, suggesting our results may not be applied to both sexes. Our study was standardised so that the right upper and lower limbs where measured, however hand dominance was not established prior to starting, and is a further limitation.

All patients met the criteria for SIRS, however not all of them were septic at the time of ultrasound

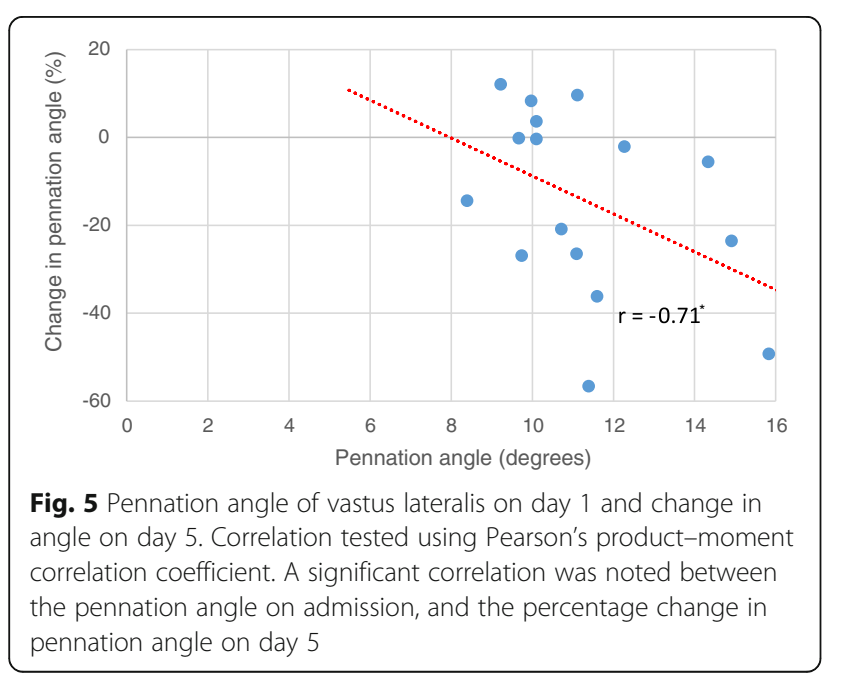

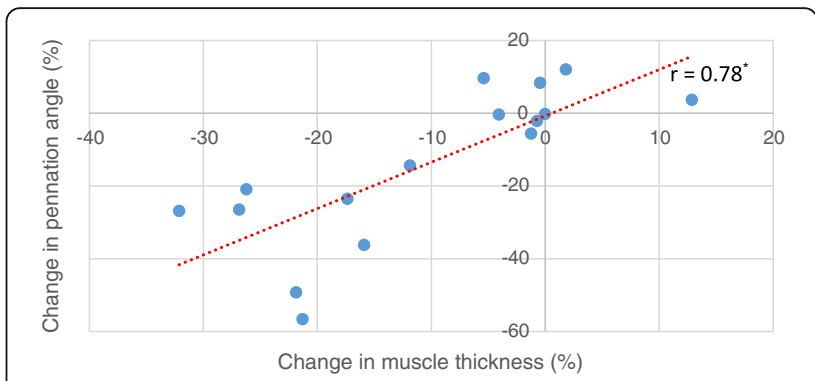

Fig. 6 Changes in muscle thickness and pennation angle on day 5 in the vastus lateralis muscle. Correlation tested using Pearson's product-moment correlation coefficient. On day 5, a significant relationship was observed between the change in muscle thickness and the change in pennation angle

scanning. Further, measurement of inflammatory markers was not undertaken at the time of scanning, nor have any muscle biopsies been taken from these patients. A larger sample would be required to compare if patients with sepsis lose more muscle compared to non-septic individuals.

\section{Conclusion}

Muscle architecture in the lower, but not in the upper limb is altered within the first five days of being intubated and mechanically ventilated, potentially reducing force generation and contributing to ICU acquired weakness. We were unable to demonstrate a change in the thickness of the muscles of the elbow flexor compartment suggesting weight bearing muscles are at greater risk of ICU acquired weakness. Monitoring of changes to muscle architecture may lead to early detection and better quantification of muscle loss compared to sole measurement of muscle thickness. Further prospective studies are required to determine if loss of pennation angle is found in the presence of a clinically diagnosed ICU acquired weakness. Interventional studies to preserve muscle function and architecture should include imaging of muscle architecture.

\section{Key messages}

- Muscle wasting occurs rapidly in the intensive care environment, but appears to preferentially affect the lower limb.

- Muscle thickness and pennation angle are lost during long term intubation and ventilation, however fascicle length appears to remain constant.

- Loss of muscle thickness is related to loss pennation angle by day 5 .

- Loss of pennation angle and muscle thickness by day 5 are related to their initial sizes on admission large muscles appear to lose more by day 5 . 


\section{Abbreviations}

FL: Fascicle length; ICUAW: Intensive Care Unit Acquired Weakness; MT: Muscle thickness; PA: Pennation angle

\section{Acknowledgements}

We would like to acknowledge Dr Jeff Little, consultant in Intensive Care, who provided support in gaining ethical approval for the project at Warrington Hospital, and to Karen Williams, research nurse at the Royal Liverpool University Hospital, who assisted with the assenting of patients.

\section{Funding}

No external sources of funding. All investigators carried out the study in the course of their clinical duties, using equipment freely available within their departments.

\section{Availability of data and materials}

The datasets supporting the conclusions of this article are available in the Zenodo repository, doi:10.5281/zenodo.47771, https://zenodo.org/record/ 47771.

\section{Authors' contributions}

PT carried out ultrasound imaging of the participants, analysed the images, performed the statistical analysis and drafted the manuscript. JT and RH both performed ultrasound imaging of the participants. JMP conceived the original study idea, participated in the design of the project and offered advice on the manuscript. IW acted as supervisor, and helped with the drafting of the manuscript and data analysis. All authors read and approved the final manuscript.

\section{Competing interests}

The authors declare that they have no competing interests.

\section{Consent for publication}

Not applicable.

\section{Ethical approval and consent to participate}

This study received ethical approval from the NHS Health Research Authority, Wales Research Ethics Committee No.4 (REC 4) in Wrexham, Wales, United Kingdom. The approval number is 13/WA/0111. This particular committee gives ethical approval to adult participants who are unable to consent themselves. In our study, assent was received that the participating patient's next of kin. In the event of their survival and discharge from ITU, patients were consented retrospectively by the unit's research nurses.

\section{Author details}

'Intensive Care Unit, Royal Liverpool University Hospital, Prescot Street, Liverpool L7 8XP, UK. ${ }^{2}$ Intensive Care Unit, Warrington General Hospital, Lovely Lane, Warrington WA5 1QG, UK. ${ }^{3}$ School of Healthcare Science, Manchester Metropolitan University, Chester Street, Manchester M15 5GD, UK. ${ }^{4}$ Institute of Aging and Chronic Disease, University of Liverpool, 4th floor, UCD Building, Daulby Streey, Liverpool L69 3GA, UK.

Received: 26 April 2016 Accepted: 13 October 2016

Published online: 29 November 2016

\section{References}

1. Kress JP, Hall JB. ICU-acquired weakness and recovery from critical illness. N Engl J Med. 2014:370:1626-35.

2. Lee CM, Fan E. ICU-acquired weakness: what is preventing its rehabilitation in critically ill patients? BMC Med. 2012;10:115

3. Kress JP, Herridge MS. Medical and economic implications of physical disability of survivorship. Semin Respir Crit Care Med. 2012;33:339-47.

4. Callahan LA, Supinski GS. Sepsis-induced myopathy. Crit Care Med. 2009;37 Suppl 1:354-67.

5. De Jonghe B, Sharshar T, Lefaucheur JP, Authier FJ, Durand-Zaleski I, Boussarsar $M$, et al. Paresis acquired in the intensive care unit: a prospective multicenter study. JAMA. 2002;288:2859-67.

6. Schefold JC, Bierbrauer J, Weber-Carstens S. Intensive care unit-acquired weakness (ICUAW) and muscle wasting in critically ill patients with severe sepsis and septic shock. J Cachex Sarcopenia Muscle. 2010;1:147-57.
7. Connolly B, MacBean V, Crowley C, Lunt A, Moxham J, Rafferty GF, et al. Ultrasound for the assessment of peripheral skeletal muscle architecture in critical illness: a systematic review. Crit Care Med. 2015;43:897-905.

8. Mueller N, Murthy S, Tainter CR, Lee J, Riddell K, Fintelmann FJ et al. Can Sarcopenia Quantified by Ultrasound of the Rectus Femoris Muscle Predict Adverse Outcome of Surgical Intensive Care Unit Patients as well as Frailty? A Prospective, Observational Cohort Study. Ann Surg. 2015. Epub ahead of print.

9. Strasser EM, Draskovits T, Praschak M, Quittan M, Graf A. Association between ultrasound measurements of muscle thickness, pennation angle, echogenicity and skeletal muscle strength in the elderly. Age (Dordr). 2013; 35:2377-88

10. Puthucheary ZA, Rawal J, McPhail M, Connolly B, Ratnayake G, Chan P, et al. Acute skeletal muscle wasting in critical illness. JAMA. 2013;310:1591-600.

11. Gruther W, Benesch T, Zorn C, Paternostro-Sluga T, Quittan M, Fialka-Moser $V$, et al. Muscle wasting in intensive care patients: ultrasound observation of the M. quadriceps femoris muscle layer. J Rehabil Med. 2008;40:185-9.

12. Kawakami Y, Abe T, Fukunaga T. Muscle-fiber pennation angles are greater in hypertrophied than in normal muscles. J Appl Physiol. 1993;74:2740-4.

13. Rutherford OM, Jones DA. Measurement of fibre pennation using ultrasound in the human quadriceps in vivo. Eur J Appl Physiol Occup Physiol. 1992:65:433-7.

14. Narici MV, Landoni $L$, Minetti AE. Assessment of human knee extensor muscle stress from in vivo physiological cross-sectional area and strength measurements. Eur J Appl Physiol. 1992;65:438-44.

15. Aagaard P, Andersen UL, Dyhre-Poulsen P, Leffers AM, Wagner A, Magnusson SP, et al. A mechanism for increased contractile strength of human pennate muscle in response to strength training: changes in muscle architecture. J Physiol. 2001;15:612-23.

16. de Boer MD, Seynnes OR, di Prampero PE, Pisot R, Mekjavić IB, Biolo G, et al. Effect of 5 weeks horizontal bed rest on human muscle thickness and architecture of weight bearing and non-weight bearing muscles. Eur J Appl Physiol. 2008:104:401-7.

17. Infantolino BW, Challis JH. Short communication: pennation angle variability in human muscle. J Appl Biomech. 2014;30:663-7.

18. Abe T, Kumagai K, Brechue WF. Muscle fascicle length is greater in sprinters than long-distance runners. Med Sci Sports Exerc. 2000;32:1125-9.

19. Nasirzade A, Ehsanbakhsh A, Ilbeygi S, Sobhkhiz A, Argavani H, Aliakbari M Relationship between sprint performance of front crawl swimming and muscle fascicle length in young swimmers. J Sports Sci Med. 2014;13:550-6.

20. Parry SM, El-Ansary D, Cartwright MS, Sarwal A, Berney S, Koopman R, et al. Ultrasonography in the intensive care setting can be used to detect changes in the quality and quantity of muscle and is related to muscle strength and function. J Crit Care. 2015;30:1151.

21. Gans C. Fiber architecture and muscle function. Exerc Sport Sci Rev. 1982:10:160-207.

22. Wisdom KM, Delp SL, Kuhl E. Review: Use it or lose it: multiscale skeletal muscle adaptation to mechanical stimuli. Biomech Model Mechanobiol. 2015;14:195-215.

23. Morse $\mathrm{Cl}$, Thom JM, Reeves ND, Birch KM, Narici MV. In vivo physiological cross-sectional area and specific force are reduced in the gastrocnemius of elderly men. J Appl Physiol. 2005;99:1050-5.

24. Reimers CD, Harder T, Saxe H. Age-related muscle atrophy does not affect all muscles and can partly be compensated by physical activity: an ultrasound study. J Neurol Sci. 1998;159:60-6.

25. Bunnell A, Ney J, Gellhorn A, Hough C. Quantitative neuromuscular ultrasound in intensive care unit acquired weakness: A systematic review. Muscle Nerve. 2015;52:701-8

26. Li Z, Peng X, Zhu B, Zhang $Y$, Xi X. Active mobilization for mechanically ventilated patients: a systematic review. Arch Phys Med Rehabil. 2013;94: 551-61.

27. Dirks ML, Hansen D, Van Assche A, Dendale P, Van Loon LJ. Neuromuscular electrical stimulation prevents muscle wasting in critically ill comatose patients. Clin Sci (Lond). 2015:128:357-65. 